\title{
Psychosocial constructs were not mediators of intervention effects for dietary and physical activity outcomes in a church-based lifestyle intervention: Delta Body and Soul III
}

\author{
Jessica L Thomson ${ }^{1, *}$, Lisa M Tussing-Humphreys ${ }^{1,2}$, Jamie M Zoellner ${ }^{3}$ and \\ Melissa H Goodman ${ }^{1}$ \\ 'US Department of Agriculture, Agricultural Research Service, 141 Experiment Station Road, Stoneville, MS 38776, \\ USA: ${ }^{2}$ Department of Medicine and Cancer Center, University of Illinois at Chicago, Chicago, IL, USA: ${ }^{3}$ Department \\ of Human Nutrition, Foods, and Exercise, Virginia Tech, Blacksburg, VA, USA
}

Submitted 27 June 2015: Final revision received 2 December 2015: Accepted 7 December 2015: First published online 22 January 2016

\begin{abstract}
Objective: Evaluating an intervention's theoretical basis can inform design modifications to produce more effective interventions. Hence the present study's purpose was to determine if effects from a multicomponent lifestyle intervention were mediated by changes in the psychosocial constructs decisional balance, self-efficacy and social support.

Design: Delta Body and Soul III, conducted from August 2011 to May 2012, was a 6-month, church-based, lifestyle intervention designed to improve diet quality and increase physical activity. Primary outcomes, diet quality and aerobic and strength/flexibility physical activity, as well as psychosocial constructs, were assessed via self-report, interviewer-administered surveys at baseline and post intervention. Mediation analyses were conducted using ordinary least squares (continuous outcomes) and maximum likelihood logistic (dichotomous outcomes) regression path analysis.

Setting: Churches (five intervention and three control) were recruited from four counties in the Lower Mississippi Delta region of the USA.

Subjects: Rural, Southern, primarily African-American adults ( $n$ 321).

Results: Based upon results from the multiple mediation models, there was no evidence that treatment (intervention $v$. control) indirectly influenced changes in diet quality or physical activity through its effects on decisional balance, self-efficacy and social support. However, there was evidence for direct effects of social support for exercise on physical activity and of self-efficacy for sugar-sweetened beverages on diet quality.

Conclusions: Results do not support the hypothesis that the psychosocial constructs decisional balance, self-efficacy and social support were the theoretical mechanisms by which the Delta Body and Soul III intervention influenced changes in diet quality and physical activity.
\end{abstract}

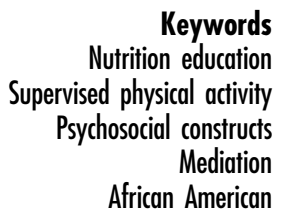

Keywords Nutrition education

Mediation

African American
The use of theoretical models in designing and implementing behavioural health interventions, including those targeting diet and exercise, has been positively associated with intervention efficacy ${ }^{(1)}$. One such theory is the Transtheoretical Model of Behaviour Change, which consists of four core constructs: stages of change, decisional balance, self-efficacy and process of change ${ }^{(2)}$. This theory postulates that for individuals to progress through behaviour change stages - precontemplation, contemplation, preparation, action and maintenance - they need a growing awareness that the advantages ('pros') of changing their behaviour outweigh the disadvantages ('cons') $^{(2)}$. This awareness is termed decisional balance, and improvements in this psychosocial construct have been associated with positive dietary changes ${ }^{(3)}$ and higher levels of physical activity ${ }^{(4)}$. Additionally, individuals require confidence in their ability to make and maintain changes in situations that tempt them to return to their old, unhealthy habits ${ }^{(2)}$. This confidence is termed self-efficacy, and higher levels of this psychosocial construct have been associated with healthy eating behaviours $^{(5-7)}$ and increased participation in exercise ${ }^{(8)}$ 
in general as well as specific populations, such as residents of rural communities ${ }^{(9)}$. Finally, covert and overt activities, also termed the cognitive and behavioural processes of change, are applied to help individuals progress through stages and maintain behavioural change. Health behaviour interventions based upon stages or processes of change have been found effective in increasing consumption of healthier foods and physical activity ${ }^{(10)}$.

When designing interventions for rural AfricanAmerican communities, social support - or the physical and emotional comfort given by family, friends and others - is an important theoretical construct to consider as it is a determinant of physical activity both in African-American adults $^{(11)}$ and residents of socio-economically disadvantaged communities ${ }^{(12)}$, as well as those living in rural settings ${ }^{(9)}$. Increased levels of social support also have been associated with higher levels of healthy eating in African-American adults ${ }^{(11)}$.

While the use of theoretically based behavioural health interventions is essential, it has become increasingly important to determine whether observed behavioural changes are supported by the psychosocial measures representing the theoretical constructs upon which the intervention was designed. For example, it may be more informative to claim that an intervention exerted its effect on diet quality via improved self-efficacy to drink less sugar-sweetened beverages (SSB) than simply showing that an intervention resulted in improved diet quality. Mediation analysis is a statistical tool that can help researchers determine how their intervention effects were achieved $^{(13)}$ and can inform design modifications (e.g. identification and refinement of critical components) to produce more effective interventions. Thus, determining whether the psychosocial constructs purported to play an important role in governing the relationship between intervention effects and changes in health behaviours are supported by study data is a crucial step in evaluating health behaviour interventions.

Such an evaluation may be particularly useful for Delta Body and Soul III, a church-based, diet and supervised physical activity intervention, which did result in increased physical activity in rural, Southern, primarily AfricanAmerican adults ${ }^{(14)}$. Additionally, significant improvements in diet quality were apparent in the intervention group and not the control group. However, differences between these two groups did not reach statistical significance. Hence, the purpose of the present study was to determine if intervention effects were mediated by changes in the psychosocial constructs decisional balance, self-efficacy and social support. While it may seem counterintuitive to test for an effect that 'does not exist', it is possible that the Delta Body and Soul III intervention exerted an effect on diet quality and physical activity indirectly through the psychosocial constructs of interest even if it cannot be established through hypothesis testing that the total intervention effect is different from zero ${ }^{(15)}$.

\section{Methods}

\section{Study design and participants}

Delta Body and Soul III is a 6-month, church-based, multicomponent intervention that was designed to improve diet quality and increase physical activity in rural, Southern, primarily African-American adults in the Lower Mississippi Delta region of the USA. Baseline data were collected between August and October 2011 and post-intervention data between March and May 2012.

Church recruitment across four Lower Mississippi Delta counties occurred via mailed study invitation letters to nine churches, followed by telephone contact to schedule an informational study presentation. Churches qualified for study participation if able to pre-register at least twenty eligible congregational members. Individual participant eligibility criteria included at least 18 years of age and not currently pregnant. A further restriction was placed on participation in the physical activity classes such that participants with baseline blood pressure greater than 160/110 mmHg or resting heart rate greater than 110 beats/ min were required to obtain written medical clearance before being allowed to participate in these classes. Of the nine churches contacted, the first five were assigned to the intervention group, the last three to the control group, and one declined to participate. More churches were intentionally assigned to the intervention to increase the statistical power for detecting changes within this group, as previous experience showed greater variability within the intervention group as compared with the control group ${ }^{(16)}$. We have reported the CONSORT (Consolidated Standards of Reporting Trials) flow diagram for this study previously ${ }^{(17)}$.

\section{Intervention}

Delta Body and Soul III was an adaptation of the original theory- and evidence-based Body and Soul programme. Body and Soul was a 6-month dietary intervention conducted through African-American churches and delivered collaboratively by community volunteers and a health-related notfor-profit agency ${ }^{(18)}$. The current study, Delta Body and Soul III, was a 6-month, church-based, multicomponent, diet and supervised physical activity intervention designed for rural Lower Mississippi Delta African-American adults. Similar to the underlying psychosocial constructs guiding the development, implementation and evaluation of the original Body and Soul, the current study targeted and assessed changes in psychosocial constructs from the Transtheoretical Model of Behavioural Change (decisional balance and self-efficacy) $)^{(2)}$ and the construct of social support ${ }^{(19)}$. Delta Body and Soul III also built upon two earlier adapted interventions of lower dose intensity conducted in the Lower Mississippi Delta region of Mississippi ${ }^{(20,21)}$.

Modifications in the current intervention included replacing peer counselling with counselling by trained research staff (telephone motivational interviewing; up to two calls). 
The motivational interviewing was conducted during months 3 through 5 of the intervention. The first motivational interviewing call consisted of developing an action plan to maintain engagement in healthy eating and physical activity. At the end of this first call, a second call was offered to participants engaging in the first call. The second call, conducted two to four weeks after the first call, was used to discuss progress related to the diet and physical activity action plan developed during the first call. For both motivational interviewing calls, a maximum of three contact attempts were made. If a participant was not reached on the third attempt, no further attempts were made. The motivational interviewing calls were approximately 15-20 min in length.

Additional modifications included broadening the dietary focus, adding three more nutrition education sessions and adding one didactic physical activity education session. Hence, the 60-min nutrition and physical activity education sessions consisted of a total of nine sessions: eight focused on nutrition and one focused on physical activity. These education sessions were held approximately every three weeks and emphasized increasing consumption of fruits, vegetables, whole grains and lowfat dairy foods; decreasing consumption of solid fats, added sugars and sodium; eating a healthy breakfast; meal planning and healthy food substitutions, including regional and cultural foods; weight and portion control; reading food labels; and childhood obesity. Presentations and activities (e.g. cooking demonstrations) were developed by the research staff and presented collaboratively with a trained church liaison who also called participants to remind them of upcoming sessions. Healthful foods and beverages consistent with lesson themes were served at these events. The single didactic physical activity session was centred on the benefit of, recommendations for and strategies for overcoming barriers to physical activity. A trained, certified fitness instructor co-led this session and taught the 60-min, semi-weekly ( $n$ 20), supervised physical activity classes which incorporated approximately equal proportions of aerobic and strength/flexibility activities. These supervised physical activity classes represented a modification from the earlier iterations of Delta Body and Soul. All study-related events were held at the churches, except in the case of the smallest church for which a nearby facility of the US Department of Agriculture was used.

Intervention participants received binders consisting of the nine educational lessons, healthy recipes and other handouts related to nutrition, chronic disease prevention and physical activity. Intervention participants also received a Delta Body and Soul cookbook and monthly newsletters that featured nutrition and physical activity topics, healthy recipes, and dates and times for upcoming education sessions and physical activity classes. Additionally, intervention participants had access to their church's health and fitness station which consisted of a digital scale, a digital blood pressure monitor, BMI and blood pressure monitoring charts, a library of culturally appropriate fitness DVDs and a television with built-in DVD player.

Participants in the control churches received bimonthly newsletters containing information pertaining to cold and influenza, food safety and minimizing stress. To compensate time for data collection procedures, \$US $30 \mathrm{gift}$ cards were provided to participants at baseline and post intervention. Further details regarding study methodology are published elsewhere ${ }^{(14)}$.

Enhancements included in the current intervention sought to inform decisional balance and improve selfefficacy, as well as increase social support for health behaviour change. Table 1 presents the intervention's individual components and subcomponents with their targeted psychosocial construct(s). Briefly, the components designed to inform decisional balance included health screenings, education, motivational interviewing, and information presented in the educational binders and monthly newsletters. The components designed to improve self-efficacy included health and fitness stations, education, physical activity classes, cookbooks featuring regional and cultural recipes, and monthly newsletters featuring participant success stories. The components designed to increase social support included the church committee and setting, kickoff event and monthly newsletters highlighting upcoming group events. Church committees were formed and led by the pastor and his wife, and typically consisted of three to five members, one of whom was usually a health professional (e.g. nurse, social

Table 1 Intervention components designed to affect psychosocial constructs: Delta Body and Soul III, Mississippi, USA, 2011-2012

\begin{tabular}{|c|c|c|c|}
\hline \multirow[b]{2}{*}{ Intervention component } & \multicolumn{3}{|c|}{ Psychosocial construct } \\
\hline & $\begin{array}{l}\text { Decisional } \\
\text { balance }\end{array}$ & $\begin{array}{c}\text { Self- } \\
\text { efficacy }\end{array}$ & $\begin{array}{l}\text { Social } \\
\text { support }\end{array}$ \\
\hline Church committee & & & $x$ \\
\hline Kickoff event & & & $\mathrm{x}$ \\
\hline Health screening & $x$ & & \\
\hline Health and fitness station & & $x$ & \\
\hline Education sessions & $\mathrm{x}$ & $x$ & $\mathrm{x}$ \\
\hline Church setting & & & $\mathrm{x}$ \\
\hline $\begin{array}{l}\text { Nutrition and PA } \\
\text { information }\end{array}$ & $x$ & & \\
\hline Meal planning & & $\mathrm{x}$ & \\
\hline Healthy food substitutions & & $x$ & \\
\hline Reading food labels & & $x$ & \\
\hline Portion size & & $\mathrm{x}$ & \\
\hline Cooking demonstrations & $x$ & & \\
\hline $\begin{array}{l}\text { Healthful foods and } \\
\text { beverages served }\end{array}$ & $x$ & & \\
\hline PA classes & & $\mathrm{x}$ & $\mathrm{x}$ \\
\hline Church setting & & & $\mathrm{x}$ \\
\hline Supervised PA & & $x$ & \\
\hline Motivational interviewing & $\mathrm{x}$ & & \\
\hline Cookbook & & $x$ & \\
\hline Educational binders & $\mathrm{x}$ & & \\
\hline Monthly newsletters & $\mathrm{x}$ & $\mathrm{x}$ & $\mathrm{x}$ \\
\hline
\end{tabular}

PA, physical activity. 
worker). The kickoff event included all five intervention churches and involved pastors signing healthful eating covenants and healthful foods prepared by a local chef.

\section{Measures}

Surveys were interviewer-administered and data included demographic characteristics (e.g. gender, race, age, marital status and employment status), self-reported medical diagnoses, smoking and psychosocial constructs (decisional balance, self-efficacy and social support). Decisional balance (pros and cons) was measured using modified versions of validated scales ${ }^{(22)}$. Self-efficacy and social support were measured using modified versions of the Self-Efficacy/Social Support and Eating Habits Surveys and the Self-Efficacy/Social Support and Exercise Habits Surveys ${ }^{(23,24)}$. Sufficient internal consistency $(0 \cdot 7 \leq$ Cronbach's $\alpha \leq 0.9$ ) was achieved at both baseline and post intervention for all scales, except decisional balance (cons) to eat fruit and resist drinking SSB and social support for exercise. Because internal consistency for fruit decisional balance (cons) and exercise social support was low only at baseline (Cronbach's $\alpha=0 \cdot 6$ ), these scales were included in the analyses. Additionally, while internal consistency for SSB decisional balance (cons) was low at both baseline and post intervention (Cronbach's $\alpha=0.6$ at both time points), this scale was included in the analyses because it had sufficient internal consistency in a previous iteration of Delta Body and Soul ${ }^{(25)}$. Nevertheless, data from these measures should be interpreted cautiously.

Dietary intake for the previous 6 months was measured using the 158-item Delta Food Frequency Questionnaire (Delta FFQ $^{(26)}$. In the validation study, energy-adjusted and de-attenuated correlations for macronutrient intakes between the Delta FFQ and $24 \mathrm{~h}$ dietary recalls ranged from 0.23 to 0.75 , while those for dietary vitamin and mineral intakes ranged from 0.24 to $0.75^{(26)}$. The Delta FFQ data were used to generate nutrient totals for energy, total fat, saturated fat, cholesterol, sodium and percentage of energy from total fat, as well as Healthy Eating Index2005 (HEI-2005) total and component scores. The HEI-2005 measures adherence to the 2005 Dietary Guidelines for Americans. The twelve components of HEI-2005 (total and whole fruit; total and dark green and orange vegetables and legumes; total and whole grains; milk; meat and beans; oils; saturated fat; sodium; and calories from solid fats, alcoholic beverages and added sugars) are summed to create a total score with a maximum value of $100^{(27)}$. For each component, higher scores reflect better adherence to recommendations of the 2005 Dietary Guidelines for Americans. Only valid ( $\leq 5$ missing questionnaire items) and plausible (daily intake between 2092 and $25104 \mathrm{~kJ}$ (500 and $6000 \mathrm{kcal}$ )) Delta FFQ were used in the analyses.

Physical activity was measured using the Rapid Assessment of Physical Activity (RAPA) ${ }^{\text {(28) }}$ which allows for classification of aerobic physical activity into one of five categories: (i) sedentary, (ii) underactive, (iii) underactive regular light, (iv) underactive regular and (v) active. Anything less than active is considered suboptimal. The RAPA also allows for classification of strength and flexibility physical activity into one of four categories: (i) none, (ii) strength only, (iii) flexibility only and (iv) both strength and flexibility. The RAPA had a comparatively high correlation ( $r=0.54)$ with the Community Healthy Activities Model Program for Seniors (CHAMPS) moderate energy expenditure and high specificity, sensitivity and positive predictive value $(62-81 \%)$ for flexibility and strength $^{(28)}$.

Anthropometric variables included height, measured using a vertical stadiometer (Shorr Production, Olney, MD, USA), and weight, measured using a calibrated digital scale (model BWB-500; Tanita Corp., Tokyo, Japan). BMI was calculated as weight (in kilograms) divided by height (in metres) squared. Blood pressure was measured using an automatic blood pressure monitor (HEM780; Omron Healthcare Inc., Kyoto, Japan). Details regarding other (e.g. clinical, medication) study measures that were not used in the current analyses can be found elsewhere ${ }^{(14)}$.

\section{Statistical analyses}

Figure 1 presents the conceptual model for mediation of intervention effects on diet quality and physical activity by the psychosocial constructs. Model coefficients $a_{1}, a_{2}$ and $a_{3}$ represent direct effects of the intervention on the psychosocial constructs. Model coefficients $b_{1}, b_{2}$ and $b_{3}$ represent direct effects of the psychosocial constructs on the diet quality and physical activity outcomes. Model coefficient $c^{\prime}$ represents the indirect effect or the proportion of the relationship between the intervention and the diet quality and physical activity outcomes that is mediated by the psychosocial constructs.

Statistical analyses were performed using the statistical software package SAS ${ }^{\circledR}$ version 9.4. Generalized linear mixed models were used to test for differences between intervention and control participants at baseline as well as for differences in outcome changes. Maximum likelihood estimation was used with group modelled as a fixed effect and church modelled as a random effect using variance components covariance matrix structure. Generalized linear mixed models were used because outcome variables were both continuous (diet quality, aerobic physical activity and psychosocial constructs) and categorical (aerobic and strength/flexibility physical activity) in nature and such models can account for both fixed and random effects. Maximum likelihood estimation is a method that can account for missing data in repeated measures. Diet quality, aerobic physical activity (continuous form) and psychosocial constructs were modelled using a Gaussian distribution with an identity link function (PROC MIXED procedure). Aerobic (categorical form) and strength/ flexibility physical activity were modelled using a binomial distribution (increase in physical activity $v$. no change or 
decrease in physical activity) with a logit link function (PROC GLIMMIX procedure). For the binomial models, overdispersion was assessed using the generalized $\chi^{2}$ statistic divided by its degrees of freedom. In all cases, this value was close to one, indicating the binomial models were correctly specified. Additionally, bivariate associations between outcome variables and predictor or explanatory variables were assessed for approximate linearity, while the residuals of all models were analysed to assess the fit of the models. Non-linear relationships were not found nor were outliers or extreme values identified.

Mediation analyses were conducted using ordinary least squares (continuous outcomes) and maximum likelihood logistic (dichotomous outcomes) regression path analysis. Path analysis was implemented using the PROCESS macro, version $2 \cdot 12 \cdot 2^{(29)}$. This macro calculated the regression coefficients for the effect of the intervention on psychosocial constructs (Fig. 1 regression coefficients $a_{1}, a_{2}$ and $a_{3}$ ), associations between changes in psychosocial constructs and changes in diet quality and physical activity (Fig. 1 regression coefficients $b_{1}, b_{2}$ and $b_{3}$ ), and direct and indirect (Fig. 1 regression coefficient $c^{\prime}$ ) intervention effects. Parallel multiple mediation models with covariates were implemented and bias-corrected bootstrap 95\% confidence intervals for the indirect effect were calculated based on 10000 bootstrap samples. Potential (parallel) mediators of intervention effects included the psychosocial constructs decisional balance (pros and cons), selfefficacy and social support. Diet quality mediation models consisted of those HEI-2005 components (total fruit, whole fruit, total vegetables, dark green and orange vegetables and legumes (DGOV\&L), whole grains, and solid fats, alcoholic beverages and added sugars (SoFAAS)) with associated psychosocial constructs (e.g. decisional

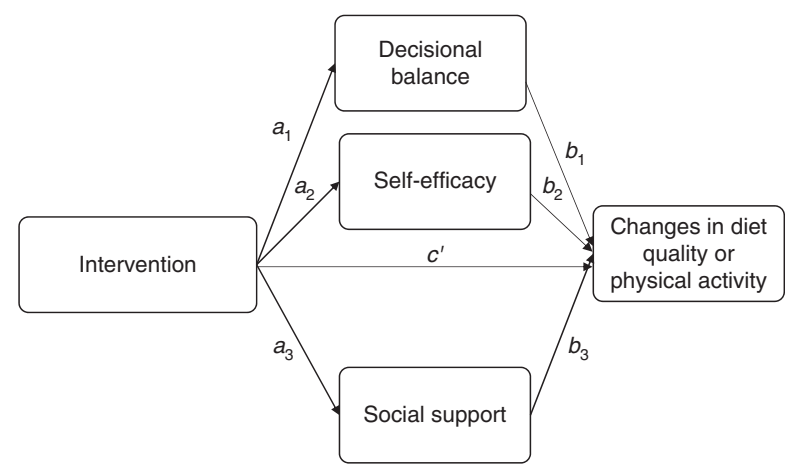

Fig. 1 Model for mediation of intervention effects on diet quality and physical activity by psychosocial constructs. Model coefficients $a_{1}, a_{2}$ and $a_{3}$ represent direct effects of the intervention on the psychosocial constructs. Model coefficients $b_{1}, b_{2}$ and $b_{3}$ represent direct effects of the psychosocial constructs on the diet quality and physical activity outcomes. Model coefficient $c^{\prime}$ represents the indirect effect or the proportion of the relationship between the intervention and the diet quality and physical activity outcomes that is mediated by the psychosocial constructs balance, self-efficacy and social support to eat fruit). Physical activity mediation models consisted of aerobic physical activity, modelled both in categorical (suboptimal $v$. optimal) and continuous (five levels) forms, and strength/flexibility physical activity, modelled in categorical form (none, some (strength or flexibility) and both (strength and flexibility)). Covariates included in these models were variables that differed between the control and intervention groups at baseline (Table 2): gender for all models; baseline strength/flexibility level for strength/ flexibility outcome changes; and decisional balance (pro) to eat fruit, to eat vegetables and to eat whole grains for corresponding dietary outcome changes. The significance level of the tests was set at $0 \cdot 05$.

\section{Results}

Retention rates for the intervention and control groups were $76 \%(219 / 287)$ and $84 \%(102 / 122)$, respectively. Table 2 shows that the majority of participants in both the control and intervention groups were female $(64 \%$ and $75 \%$, respectively), African American (98\% for both groups), non-smokers ( $80 \%$ and $86 \%$, respectively) and owned a vehicle (70\% and $82 \%$, respectively). Mean BMI was 35 and $33 \mathrm{~kg} / \mathrm{m}^{2}$ in the intervention and control groups, respectively, while mean age was 47 years for both groups. Differences apparent between the control and intervention participants at baseline included a higher proportion of females in the intervention group, a higher proportion of participants engaging in some strength/flexibility physical activity in the control group, and higher scores for decisional balance (pros) to eat fruit, vegetables and whole grains in the intervention group. Nineteen $(7 \%)$ intervention participants were ineligible to partake in the physical activity classes due to failure to obtain medical clearance.

\section{Changes in diet quality, physical activity and psychosocial constructs}

Table 3 presents the results of the tests for significant within- and between-group changes. Significant increases in HEI-2005 total fruit, whole fruit, total vegetable, DGOV\&L and SoFAAS scores were observed in the intervention group (range 0.4 to 1.3 points). Although no changes were significant in the control group, significant between-group differences were not found. The significant decrease in aerobic physical activity observed in the control group was not significantly different from the nonsignificant decrease observed in the intervention group. However, for those intervention participants reporting changes in physical activity levels from baseline to post intervention, significantly more participants reported increased strength/flexibility activity (28\%) as compared with decreased activity $(12 \%, P<0 \cdot 001)$. Conversely, for those control participants reporting changes in activity 
Table 2 Baseline characteristics for and comparisons between Delta Body and Soul III control $(n$ 122) and intervention participants $(n$ 287), Mississippi, USA, 2011-2012

\begin{tabular}{|c|c|c|c|c|c|c|}
\hline \multirow[b]{2}{*}{ Characteristic } & & \multicolumn{2}{|c|}{ Control } & \multicolumn{2}{|c|}{ Intervention } & \multirow[b]{2}{*}{$P$} \\
\hline & & $n$ & $\%$ & $n$ & $\%$ & \\
\hline Female & & 78 & $63 \cdot 9$ & 216 & $75 \cdot 3$ & 0.014 \\
\hline African American* & & 120 & 98.4 & 282 & $98 \cdot 3$ & 0.739 \\
\hline Married/living with significant other† & & 55 & 45.5 & 129 & $45 \cdot 1$ & 0.924 \\
\hline > High school education & & 50 & $41 \cdot 7$ & 163 & $56 \cdot 8$ & 0.157 \\
\hline Employedł & & 56 & $46 \cdot 7$ & 154 & $54 \cdot 6$ & 0.247 \\
\hline Health insurance & & 85 & $72 \cdot 6$ & 196 & $72 \cdot 3$ & 0.938 \\
\hline Own vehicle & & 85 & $69 \cdot 7$ & 235 & $81 \cdot 9$ & 0.089 \\
\hline Smoker & & 24 & 19.7 & 40 & 13.9 & 0.182 \\
\hline \multicolumn{7}{|l|}{ Chronic health condition } \\
\hline Diabetes & & 25 & $21 \cdot 0$ & 59 & 21.3 & 0.897 \\
\hline Hypertension & & 65 & 53.7 & 154 & $54 \cdot 0$ & 0.760 \\
\hline High cholesterol & & 33 & $27 \cdot 7$ & 63 & $22 \cdot 3$ & 0.291 \\
\hline \multicolumn{7}{|l|}{ Physical activity } \\
\hline Optimal aerobic & & 41 & 33.9 & 102 & $36 \cdot 4$ & 0.643 \\
\hline \multirow[t]{2}{*}{ Some strength/flexibility } & & 61 & $50 \cdot 8$ & 105 & $37 \cdot 5$ & 0.013 \\
\hline & Scale range & Mean & SD & Mean & SD & $P$ \\
\hline Age (years) & - & $47 \cdot 0$ & $16 \cdot 40$ & $47 \cdot 3$ & $14 \cdot 43$ & 0.140 \\
\hline $\mathrm{BMl}\left(\mathrm{kg} / \mathrm{m}^{2}\right)$ & - & $32 \cdot 6$ & 8.24 & 34.5 & 8.33 & 0.152 \\
\hline Systolic blood pressure (mmHg) & - & $135 \cdot 1$ & 22.56 & $132 \cdot 6$ & 19.54 & 0.364 \\
\hline Diastolic blood pressure $(\mathrm{mmHg})$ & - & $82 \cdot 0$ & 11.66 & $78 \cdot 7$ & 11.35 & 0.142 \\
\hline \multicolumn{7}{|l|}{ Healthy Eating Index-2005 } \\
\hline Total fruit & $0-5$ & $2 \cdot 6$ & 1.52 & $2 \cdot 4$ & 1.49 & 0.473 \\
\hline Whole fruit & $0-5$ & $2 \cdot 2$ & 1.55 & $2 \cdot 4$ & 1.53 & 0.321 \\
\hline Total vegetables & $0-5$ & 3.4 & $1 \cdot 19$ & $3 \cdot 2$ & 1.29 & 0.394 \\
\hline DGOV\&L & $0-5$ & $2 \cdot 8$ & 1.51 & $2 \cdot 4$ & 1.52 & 0.083 \\
\hline Whole grain & $0-5$ & 1.5 & 1.43 & $2 \cdot 0$ & 1.66 & 0.182 \\
\hline SoFAAS & $0-20$ & $12 \cdot 2$ & $5 \cdot 14$ & $12 \cdot 1$ & 4.50 & 0.804 \\
\hline Total & $0-100$ & $56 \cdot 1$ & 11.03 & $55 \cdot 8$ & $10 \cdot 16$ & 0.892 \\
\hline \multicolumn{7}{|l|}{ Psychosocial constructs } \\
\hline DB (pro) - fruit & $5-20$ & $16 \cdot 6$ & $3 \cdot 70$ & $17 \cdot 6$ & $2 \cdot 21$ & 0.027 \\
\hline DB (con) - fruit & $6-24$ & $16 \cdot 0$ & 3.23 & $15 \cdot 4$ & $3 \cdot 10$ & 0.163 \\
\hline SE - fruit & $4-16$ & $12 \cdot 2$ & 2.69 & $12 \cdot 2$ & $2 \cdot 76$ & 0.730 \\
\hline SS - fruit & $9-36$ & $25 \cdot 8$ & $4 \cdot 21$ & $25 \cdot 6$ & 4.21 & 0.668 \\
\hline DB (pro) - vegetable & $5-20$ & $16 \cdot 8$ & $3 \cdot 16$ & $17 \cdot 6$ & $2 \cdot 39$ & 0.046 \\
\hline DB (con) - vegetable & $6-24$ & $16 \cdot 0$ & 3.31 & $16 \cdot 3$ & 3.35 & 0.529 \\
\hline SE - vegetable & $4-16$ & 11.7 & 3.06 & $12 \cdot 2$ & $2 \cdot 87$ & 0.222 \\
\hline SS - vegetable & $9-36$ & $26 \cdot 1$ & 4.44 & $26 \cdot 4$ & 4.27 & 0.627 \\
\hline DB (pro) - whole grains & $5-20$ & $16 \cdot 2$ & 3.34 & $17 \cdot 1$ & $2 \cdot 71$ & 0.027 \\
\hline DB (con) - whole grains & $4-16$ & $10 \cdot 2$ & 2.54 & $10 \cdot 1$ & 2.56 & 0.832 \\
\hline SE - whole grains & $4-16$ & $10 \cdot 7$ & 3.00 & 11.2 & 2.67 & 0.250 \\
\hline SS - whole grains & $9-36$ & $24 \cdot 1$ & 4.50 & $23 \cdot 8$ & 4.09 & 0.609 \\
\hline DB (pro) - SSB & $4-16$ & $7 \cdot 4$ & 1.91 & 7.9 & 2.00 & 0.098 \\
\hline SE - SSB & $4-16$ & 9.4 & 2.51 & $10 \cdot 0$ & 2.66 & 0.089 \\
\hline$S S-S S B$ & $5-20$ & $12 \cdot 9$ & $3 \cdot 19$ & $12 \cdot 4$ & 3.23 & 0.317 \\
\hline DB (pro) - exercise & $4-16$ & 13.5 & $2 \cdot 30$ & $13 \cdot 9$ & $2 \cdot 00$ & 0.134 \\
\hline DB (con) - exercise & $4-16$ & 11.2 & 2.38 & 11.0 & 2.57 & 0.600 \\
\hline SE - exercise & $6-24$ & $16 \cdot 2$ & 3.50 & $16 \cdot 5$ & 2.92 & 0.513 \\
\hline SS - exercise & $10-40$ & 28.6 & 4.05 & 28.2 & 3.76 & 0.400 \\
\hline
\end{tabular}

DGOV\&L, dark green and orange vegetables and legumes; SoFAAS, solid fats, alcoholic beverages and added sugars; DB, decisional balance; SE, self-efficacy; SS, social support; SSB, sugar-sweetened beverages.

${ }^{*}$ Other category included white, Hispanic, other and multiracial.

†Not married category included never married, widowed, divorced and separated.

‡Employed (full time, part time or self-employed) $v$. unemployed (unemployed, retired, student or disabled).

levels from baseline to post intervention, significantly more participants reported decreased optimal aerobic and strength/flexibility activity ( $23 \%$ and $28 \%$, respectively) as compared with increased activity (7\% and $12 \%$, respectively; $P=0.004$ and 0.001). Significant increases in all social support scales as well as self-efficacy to resist drinking SSB were observed in the intervention group (range 0.8 to 1.7 points). Similarly, significant increases in fruits and vegetables social support, whole grains decisional balance (pros) and SSB self-efficacy were observed in the control group (range 0.9 to 1.3 points). Significant between-group differences were not found for any decisional balance, self-efficacy or social support scales (Fig. 1 regression coefficients $a_{1}, a_{2}$ and $a_{3}$, respectively). 
Table 3 Dietary, physical activity and psychosocial outcome changes for and comparisons between Delta Body and Soul III control ( $n$ 102) and intervention participants ( $n$ 219), Mississippi, USA, 2011-2012

\begin{tabular}{|c|c|c|c|c|c|c|}
\hline \multirow[b]{2}{*}{ Characteristic } & \multirow[b]{2}{*}{ Scale range } & \multicolumn{2}{|c|}{ Control } & \multicolumn{2}{|c|}{ Intervention } & \multirow[b]{2}{*}{$P$} \\
\hline & & LSM & SEM & LSM & SEM & \\
\hline \multicolumn{7}{|l|}{ Healthy Eating Index-2005 } \\
\hline Total fruit & $0-5$ & 0.1 & 0.21 & 0.5 & 0.15 & 0.180 \\
\hline Whole fruit & $0-5$ & 0.2 & 0.26 & 0.5 & 0.19 & 0.446 \\
\hline Total vegetables & $0-5$ & 0.2 & 0.18 & 0.4 & 0.12 & 0.493 \\
\hline DGOV\&L & $0-5$ & 0.2 & 0.23 & 0.6 & 0.17 & 0.197 \\
\hline Whole grain & $0-5$ & -0.1 & 0.18 & 0.2 & 0.12 & 0.174 \\
\hline SoFAAS & $0-20$ & 0.2 & 0.68 & 1.3 & 0.49 & 0.256 \\
\hline Aerobic physical activity & $1-5$ & -0.6 & 0.16 & -0.1 & 0.11 & 0.061 \\
\hline \multicolumn{7}{|l|}{ Psychosocial constructs } \\
\hline DB (pro) - fruit & $5-20$ & 0.7 & 0.43 & 0.0 & 0.32 & 0.271 \\
\hline DB (con) - fruit & $6-24$ & -0.9 & 0.37 & -0.4 & 0.26 & 0.272 \\
\hline SE - fruit & $4-16$ & 0.2 & 0.44 & -0.1 & 0.32 & 0.608 \\
\hline SS - fruit & $9-36$ & $1 \cdot 3$ & 0.48 & 1.6 & 0.33 & 0.620 \\
\hline DB (pro) - vegetable & $5-20$ & 0.6 & 0.32 & 0.2 & 0.23 & 0.388 \\
\hline DB (con) - vegetable & $6-24$ & -0.8 & 0.39 & -0.1 & 0.26 & 0.176 \\
\hline SE - vegetable & $4-16$ & 0.6 & 0.35 & 0.5 & 0.25 & 0.780 \\
\hline SS - vegetable & $9-36$ & $1 \cdot 3$ & 0.50 & 1.2 & 0.34 & 0.828 \\
\hline DB (pro) - whole grains & $5-20$ & 0.9 & 0.33 & 0.6 & 0.23 & 0.395 \\
\hline DB (con) - whole grains & $4-16$ & -0.4 & 0.31 & 0.3 & 0.21 & 0.098 \\
\hline SE - whole grains & $4-16$ & 0.2 & 0.46 & 0.2 & 0.32 & 0.983 \\
\hline SS - whole grains & $9-36$ & $1 \cdot 1$ & 0.51 & 1.1 & 0.37 & 0.970 \\
\hline DB (pro) - SSB & $4-16$ & 0.2 & 0.23 & 0.2 & 0.17 & 0.993 \\
\hline SE - SSB & $4-16$ & 0.9 & 0.31 & 0.8 & 0.22 & 0.924 \\
\hline SS - SSB & $5-20$ & 0.3 & 0.35 & $1 \cdot 1$ & 0.25 & 0.103 \\
\hline DB (pro) - exercise & $4-16$ & 0.6 & 0.25 & 0.2 & 0.18 & 0.210 \\
\hline DB (con) - exercise & $4-16$ & 0.1 & 0.30 & 0.4 & 0.21 & 0.413 \\
\hline SE - exercise & $6-24$ & 0.6 & 0.39 & -0.2 & 0.26 & 0.151 \\
\hline SS - exercise & $10-40$ & 0.2 & 0.52 & 1.7 & 0.36 & 0.055 \\
\hline
\end{tabular}

LSM, least squares mean (bold values indicate that within-group change is significant); SEM, standard error of mean; DGOV\&L, dark green and orange vegetables and legumes; SoFAAS, solid fats, alcoholic beverages and added sugars; DB, decisional balance; SE, self-efficacy; SS, social support; SSB, sugar-sweetened beverages.

\section{Assessment of mediation of intervention effects on diet quality and physical activity by psychosocial constructs}

Based upon mediation model results, there was no evidence that treatment (intervention $v$. control) indirectly influenced changes in diet quality (total fruit, whole fruit, total vegetables, DGOV\&L, whole grains, SoFAAS and total) or physical activity (aerobic and strength/flexibility) through its effects on decisional balance, self-efficacy and social support (see online supplementary material, Supplemental Figs 1-12 regression coefficient $c^{\prime} ; 95 \%$ CI contained zero for all indirect model coefficients). However, SSB self-efficacy directly influenced changes in SoFAAS diet quality (Supplemental Fig. 6 regression coefficient $\left.b_{2}=0.30,95 \% \mathrm{CI} 0.07,0.52\right)$ and total diet quality (Supplemental Fig. 8 regression coefficient $b_{2}=0.57,95 \%$ CI $0 \cdot 12,1 \cdot 02$ ). Similarly, social support to eat whole grains directly influenced changes in total diet quality (Supplemental Fig. 9 regression coefficient $b_{3}=0.37,95 \%$ CI $0 \cdot 04,0 \cdot 70$ ). Finally, social support for exercise directly influenced changes in both aerobic (optimal) (Supplemental Fig. 11 regression coefficient $b_{3}=0.09,95 \%$ CI $\left.0.01,0.17\right)$ and strength/flexibility physical activity (Supplemental Fig. 12 regression coefficient $b_{3}=0.09,95 \%$ CI $\left.0.01,0 \cdot 18\right)$.

\section{Discussion}

Previously published primary analysis results from Delta Body and Soul III indicated that the intervention was effective in increasing physical activity in this cohort of Southern African-American adults ${ }^{(14)}$. Additionally, while group differences were not apparent for diet quality changes, improvements in the intervention group were both statistically significant and clinically meaningful. Hence, the objective of the current secondary analysis was to determine if the psychosocial constructs decisional balance (pros and cons), self-efficacy and social support mediated intervention effects on diet quality and physical activity. Results from the present analyses provide little evidence that the intervention induced changes in the psychosocial constructs, other than social support. In comparison, intervention-induced changes in self-efficacy, but not decisional balance (with the exception of vegetables) or social support, were apparent in a previous iteration of the intervention, Delta Body and Soul $\mathrm{II}^{(25)}$. The conflicting results from these two similarly designed studies conducted in the same population may be the result of design modifications in the present study that appeared effective for increasing social support but perhaps less so for self-efficacy and decisional balance. 
Additionally, results may differ between the two studies because of analytical issues. In the present study, control and intervention groups were compared with one another, while in Delta Body and Soul II, three groups were compared with one another: control, low attendance intervention and high attendance intervention ${ }^{(25)}$.

The current study results also do not support the hypothesis that the observed changes in diet quality and physical activity outcomes were mediated by changes in decisional balance, self-efficacy or social support. Support for mediation effects of self-efficacy and social support on diet changes, specifically fruit and vegetable consumption, can be found in the original Body and Soul effectiveness $\operatorname{trial}^{(30)}$. Likewise, mediation effects of self-efficacy and social support on diet changes (dietary fat, fibre, and fruit and vegetable intakes) were reported for Guide to Health, a church-based, Internet intervention ${ }^{(31)}$. Guide to Health included twelve online modules targeting social support (e.g. getting someone to remind you to walk, adding healthier foods in ways acceptable to family members), self-efficacy (i.e. guided, gradual behaviour change) and outcome expectations (i.e. providing feasible and acceptable strategies for healthy eating and exercise) ${ }^{(31)}$. In contrast, no evidence for mediation effects of church support, self-efficacy and exercise enjoyment on increased physical activity was found in Health-e-AME, a theorybased physical activity intervention targeting Southern African-American Episcopal churches ${ }^{(32)}$. Additionally, no evidence for mediation effects of self-efficacy, social support and church support on leisure-time physical activity and fruit and vegetable consumption was found in the Faith, Activity, and Nutrition programme, another theory-based intervention targeting Southern AfricanAmerican Episcopal churches ${ }^{(33)}$. Taken together, results from these studies suggest that the importance of decisional balance, self-efficacy and social support as mediators for behavioural health intervention effects remains unclear. It may be, given the array of health interventions conducted, that the potential for mediation of intervention effects by psychosocial constructs is specific to the intervention setting, the behaviours targeted for change and the population chosen for intervention.

While no mediation effects were apparent for the psychosocial measures in the present study, evidence supporting direct effects for SSB self-efficacy on changes in SoFAAS diet quality and for exercise social support on changes in physical activity was found. In comparison, social support to eat vegetables was a significant predictor of total vegetable diet quality and decisional balance (pro) to resist drinking SSB was a significant predictor of SoFAAS diet quality in Delta Body and Soul II. However, these were not results of a mediation analysis, but rather a regression analysis. Others also have reported positive associations between self-efficacy and healthy eating behaviours $^{(5-7,30)}$, as well as between social support and physical activity ${ }^{(12,34-36)}$. Hence, while results from the current study did not support indirect effects for the psychosocial constructs, they did support direct effects for self-efficacy and social support on specific health outcomes.

The general lack of indirect and direct effects for the psychosocial constructs decisional balance, self-efficacy and social support apparent in the current study may be partially explained by the general lack of an intervention effect on these measures. Changes in decisional balance were small in magnitude, in the non-hypothesized direction (i.e. decrease) or significant for the control but not the intervention group. Similar results were observed for changes in self-efficacy, with the exception of SSB for which significant positive changes were apparent, albeit in both the control and intervention groups. While increases in social support were observed for all four of the diet quality components and physical activity in the intervention group, only social support for exercise had a significant direct effect. This may be due to the fact that the change in this measure was small and non-significant for the control group, whereas for the other social support measures, the magnitudes of changes were similar between control and intervention groups. Given that the current study represents the third iteration of Delta Body and Soul conducted in the same region of the Mississippi Delta, it is likely that diffusion of the project's goals and methods occurred and may have affected the control churches' social network as well as control participants' intentions for making diet and physical activity changes. Additionally, even in the absence of active intervention, social support from church friends for healthy eating and physical activity was found to be fairly common in rural Southern churches ${ }^{(37)}$.

The present study had several strengths including intervention in a community with elevated chronic disease prevalence, inclusion of both male and female AfricanAmerican adults, incorporation of evidence-based intervention components grounded in behaviour theory ${ }^{(18)}$ and the use of a more modern path analysis approach for mediation analysis (i.e. not a causal steps approach $)^{(15)}$. Validated measures were used for all dietary, physical activity and psychosocial variables. However, the ceiling effect for the RAPA tool is a limitation of the physical activity data as increases in activity could not be determined for participants achieving the highest levels at baseline as well as decreases for participants in the lowest levels. Although processes of change are a core component of the Transtheoretical Model of Behaviour Change, changes in behavioural and cognitive processes were not measured. This was a pragmatic decision based upon the lack of suitable measures to assess process of change for each of the targeted dietary outcomes, the fact that the study was not intended to be a stage-matched intervention and reduction of participant burden. Additionally, it is possible we did not assess the appropriate psychosocial mediators or that the timing of survey administration was 
not sufficient to detect changes. Measuring changes in psychosocial mediators at study end may have captured participants' awareness of the impending challenge of maintaining diet and physical activity changes on their own ${ }^{(38)}$. The self-report nature of these outcomes and the associated potential for recall bias and provision of socially desirable responses may be viewed as a study limitation. The relatively large number of statistical tests performed for group differences in psychosocial measures may have resulted in spurious results. However, because our mediators were chosen in advance on the basis of theory and prior empirical research, our analysis was likely less susceptible to chance findings than it might have been had we selected our mediators after the study was conducted $^{(39)}$. Generalizability of the results is limited because our participants were primarily African-American residents of rural Southern communities. Finally, it is possible that our sample size was insufficient to detect mediation. However, based on empirical estimates of required sample sizes and assuming path coefficient sizes between small and medium, our sample size had sufficient power to detect the presence of mediation ${ }^{(40)}$.

\section{Conclusion}

In conclusion, despite using a theory-based design, the psychosocial constructs targeted and measured in Delta Body and Soul III - decisional balance, self-efficacy and social support - did not appear to be the mechanisms by which this intervention influenced changes in diet quality and physical activity. The general lack of intervention effect on decisional balance and self-efficacy suggests that the intervention's components specifically targeting these constructs were ineffectual and hence may need to be strengthened. In terms of social support, evidence supporting a direct effect was present, but little evidence existed supporting an indirect effect, suggesting that the majority of the intervention effects occurred through a mechanism other than social support. The present study highlights the importance of moving beyond simply establishing an intervention's efficacy to determining the aspects of an intervention that contribute to the changes observed ${ }^{(13)}$.

\section{Acknowledgements}

Acknowledgements: The authors gratefully acknowledge the original investigators who developed the intervention, the Delta Body and Soul research team, Delta Health Alliance and the church leaders, committee members and study participants for their tremendous support. Financial support: This work was supported by the US Department of Agriculture, Agricultural Research Service (project number 6401-51000-001-00D); and the US Department of Health and Human Services, Health Resources Services Administration (grant number 6 U1FRHA07411). The views expressed are solely those of the authors and do not reflect the official policy or position of the US government. Conflict of interest: None. Authorship: J.L.T. conducted the data analysis, interpreted the data, contributed to the intellectual content of the manuscript and wrote the manuscript. L.M.T-H. designed and obtained funding for the study, was responsible for data collection and processing, contributed to the intellectual content of the manuscript and reviewed the final manuscript. J.M.Z. and M.H.G. contributed to the intellectual content of the manuscript and reviewed the final manuscript. Ethics of buman subject participation: This study was conducted according to the guidelines laid down in the Declaration of Helsinki and all procedures involving human subjects were approved by the Institutional Review Board of Delta State University, Cleveland, Mississippi, USA. Written informed consent was obtained from all subjects.

\section{Supplementary material}

To view supplementary material for this article, please visit http://dx.doi.org/10.1017/S1368980015003602

\section{References}

1. Jepson R, Harris F, MacGillivray S et al. (2006) A Review of the Effectiveness of Interventions, Approaches and Models at Individual, Community and Population Level That Are Amied at Changing Health Outcomes Through Changing Knowledge, Attitudes and Behavior. Stirling/Dundee: Cancer Care Research Centre, University of Stirling and Alliance for Self Care Research, University of Abertay.

2. Prochaska JO, DiClemente CC \& Norcross JC (1992) In search of how people change. Applications to addictive behaviors. Am Psychol 47, 1102-1114.

3. Salehi L, Mohammad K \& Montazeri A (2011) Fruit and vegetables intake among elderly Iranians: a theory-based interventional study using the five-a-day program. Nutr J 10, 123.

4. Conroy MB, Simkin-Silverman LR, Pettee KK et al. (2007) Lapses and psychosocial factors related to physical activity in early postmenopause. Med Sci Sports Exerc 39, 1858-1866.

5. Anderson ES, Winett RA \& Wojcik JR (2007) Self-regulation, self-efficacy, outcome expectations, and social support: social cognitive theory and nutrition behavior. Ann Behav Med 34, 304-312.

6. Folta SC, Lichtenstein AH, Seguin RA et al. (2009) The StrongWomen-Healthy Hearts program: reducing cardiovascular disease risk factors in rural sedentary, overweight, and obese midlife and older women. Am J Public Health 99, 1271-1277.

7. Luszczynska A \& Cieslak R (2009) Mediated effects of social support for healthy nutrition: fruit and vegetable intake across 8 months after myocardial infarction. Behav Med $\mathbf{3 5}$, $30-38$.

8. Rhodes RE \& Yao CA (2015) Models accounting for intention-behavior discordance in the physical activity domain: a user's guide, content overview, and review of current evidence. Int J Behav Nutr Phys Act 12, 9.

9. Kegler MC, Swan DW, Alcantara I et al. (2014) The influence of rural home and neighborhood environments on healthy eating, physical activity, and weight. Prev Sci 15, $1-11$. 
10. Fleig L, Kuper C, Lippke S et al. (2015) Cross-behavior associations and multiple health behavior change: a longitudinal study on physical activity and fruit and vegetable intake. J Health Psychol 20, 525-534.

11. Watters JL, Satia JA \& Galanko JA (2007) Associations of psychosocial factors with fruit and vegetable intake among African-Americans. Public Health Nutr 10, 701-711.

12. Debnam K, Holt CL, Clark EM et al. (2012) Relationship between religious social support and general social support with health behaviors in a national sample of African Americans. J Behav Med 35, 179-189.

13. Lockwood CM, DeFrancesco CA, Elliot DL et al. (2010) Mediation analyses: applications in nutrition research and reading the literature. J Am Diet Assoc 110, 753-762.

14. Thomson JL, Goodman MH \& Tussing-Humphreys L (2015) Diet quality and physical activity outcome improvements resulting from a church-based diet and supervised physical activity intervention for rural, Southern, African American adults: Delta Body and Soul III. Health Promot Pract 16 677-688.

15. Hayes AF (2013) What about Baron and Kenny? In Introduction to Mediation, Moderation, and Conditional Process Analysis, pp. 166-172. New York: Guilford Press.

16. Dumville JC, Hahn S, Miles JN et al. (2006) The use of unequal randomisation ratios in clinical trials: a review. Contemp Clin Trials 27, 1-12.

17. Thomson JL, Tussing-Humphreys LM, Goodman MH et al. (2015) Engagement indicators predict health changes in a lifestyle intervention. Am J Health Behav 39, 409-420.

18. Resnicow K, Campbell MK, Carr C et al. (2004) Body and soul. A dietary intervention conducted through AfricanAmerican churches. Am J Prev Med 27, 97-105.

19. Israel BA \& McLeroy KR (1985) Social networks and social support: implications for health education. Introduction. Health Educ Q 12, 1-4.

20. Tussing-Humphreys LM, Thomson JL, Mayo T et al. (2013) A church-based diet and physical activity intervention for rural, Lower Mississippi Delta African American adults: Delta Body and Soul effectiveness study, 2010-2011. Prev Chronic Dis 10, E92.

21. Tussing-Humphreys LM, Thomson JL \& Onufrak SJ (2015) A church-based pilot study designed to improve dietary quality for rural, lower Mississippi Delta, African American adults. J Relig Health 54, 455-469.

22. De Vet E, de Nooijer J, de Vries NK et al. (2006) The transtheoretical model for fruit, vegetable and fish consumption: associations between intakes, stages of change and stage transition determinants. Int J Behav Nutr Phys Act 3, 13.

23. Sallis JF, Grossman RM, Pinski RB et al. (1987) The development of scales to measure social support for diet and exercise behaviors. Prev Med 16, 825-836.

24. Sallis JF, Pinski RB, Grossman RM et al. (1988) The development of self-efficacy scales for health-related diet and exercise behaviors. Health Educ Res 3, 283-292.

25. Thomson JL, Zoellner JM \& Tussing-Humphreys LM (2014) Psychosocial predictors of diet and physical activity in African-Americans: results from the Delta Body and
Soul effectiveness trial, 2010-2011. Am J Health Promot 28, e81-e91.

26. Carithers TC, Talegawkar SA, Rowser ML et al. (2009) Validity and calibration of food frequency questionnaires used with African-American adults in the Jackson Heart Study. J Am Diet Assoc 109, 1184-1193.

27. Guenther PM, Reedy J \& Krebs-Smith SM (2008) Development of the Healthy Eating Index-2005. J Am Diet Assoc 108, 1896-1901.

28. Topolski TD, LoGerfo J, Patrick DL et al. (2006) The Rapid Assessment of Physical Activity (RAPA) among older adults. Prev Chronic Dis 3, A118.

29. Hayes AF (2014) PROCESS macro for SAS, V2.12.2. http:// www.afhayes.com/spss-sas-and-mplus-macros-and-code.html (accessed September 2014).

30. Fuemmeler BF, Masse LC, Yaroch AL et al. (2006) Psychosocial mediation of fruit and vegetable consumption in the body and soul effectiveness trial. Health Psychol 25, 474-483.

31. Anderson ES, Winett RA, Wojcik JR et al. (2010) Social cognitive mediators of change in a group randomized nutrition and physical activity intervention: social support, self-efficacy, outcome expectations and self-regulation in the guide-to-health trial. J Health Psychol 15, 21-32.

32. Baruth M, Wilcox S, Blair S et al. (2010) Psychosocial mediators of a faith-based physical activity intervention: implications and lessons learned from null findings. Health Educ Res 25, 645-655.

33. Baruth M \& Wilcox S (2015) Psychosocial mediators of physical activity and fruit and vegetable consumption in the Faith, Activity, and Nutrition programme. Public Health Nutr 18, 2242-2250.

34. Baird J, Cooper C, Margetts BM et al. (2009) Changing health behaviour of young women from disadvantaged backgrounds: evidence from systematic reviews. Proc Nutr SOC 68, 195-204.

35. Cleland CL, Tully MA, Kee F et al. (2012) The effectiveness of physical activity interventions in socio-economically disadvantaged communities: a systematic review. Prev Med 54, 371-380.

36. George ES, Kolt GS, Duncan MJ et al. (2012) A review of the effectiveness of physical activity interventions for adult males. Sports Med 42, 281-300.

37. Kegler MC, Escoffery C, Alcantara IC et al. (2012) Perceptions of social and environmental support for healthy eating and physical activity in rural southern churches. $J$ Relig Health 51, 799-811.

38. McAuley E, Mailey EL, Mullen SP et al. (2011) Growth trajectories of exercise self-efficacy in older adults: influence of measures and initial status. Health Psychol 30, 75-83.

39. Mackinnon DP, Cheong J \& Pirlott AG (2012) Statistical mediation analysis. In APA Handbook of Research Methods in Psychology, vol. 2, pp. 313-332 [H Cooper, editor]. Washington, DC: American Psychological Association.

40. Fritz MS \& Mackinnon DP (2007) Required sample size to detect the mediated effect. Psychol Sci 18, 233-239. 